\title{
The Wazewski topological method for contingent equations (*).
}

\author{
J. W. BEBERNes and J.D. SchuUR (U.S.A.) (**)
}

\begin{abstract}
Summary. - A Wazewski - type theorem for contingent equations is obtained using the fun. damental theory of contingent equations.
\end{abstract}

\section{1. - Introduction.}

The topological method of WAZEwsKI [12], which was originally stated for ordinary differential equations whose solutions are unique with respect to initial conditions, has been subsequently extended to equations without uniqueness (see for example, Bielecki [4], Jackson and Krassen [9], or BEbkRNes and ScHudr [3]) and to contingent equations (see for example, BiELECKI and KLUCZNy [5], or KLdCZNy [10]).

KuUCzNY discusses several of these extensions. And in his paper he shows that if a family of curves satisfies certain postulates, then a WAZEWSKI theorem may be proved for this family. By proving that the family of solutions of a contingent equation satisfies these postulates, it the follows that a WA ZEWSKI theorem holds.

Here we develop the fundamental theory of contingent equations along lines suggested by YoRke [14], [15]. We then use this theory to study the properties of a set-valued consequent mapping and to obtain a WAZEWSKI theorem for contingent equations.

Let $c\left(R^{n}\right)\left(c c\left(R^{n}\right)\right)$ denote the family of all nonempty compact (compact and convex) subsets of $R^{n}$. For $x \in R^{n}$ and $A, B \in c\left(R^{n}\right)$, let $|x|$ be the Euclidean norm, $r(x, B)=\inf \{|x-y|: y \in B\}, r(A, B)=\sup \{r(x, B): x \in A\}$, and $d(A, B)=\max \{r(A, B), r(B, A)\}$. Let $N_{a}(A)=\{x: r(x, A)<a\}$.

Let $R^{1} \times R^{n}=W$ and denote points of $W$ by $P=\left(t_{p}, x_{p}\right)$, or just $(t, x)$, or by $P_{n}=\left(t_{n}, x_{n}\right)$. For $P, Q \in W,|P-Q|$ is the Euclidean norm. For $A \subset W$ $\operatorname{Fr}(A)$ is the frontier of $A$ and $\bar{A}$ is the closure of $A$.

(*) This work was done at the Istituto Matematico dell' Universita di Firenze under the auspices of the Italian Research Council (C. N. R.). The resident addresses of the authors are, respectively, the University of Colorado, Boulder, Colo, U.S.A. and Michigan State University, East Lansing, Mich., U. S. A.

(**) Entrata in Redazione il 20 febbraio 1970. 
For $E \subset W$ and $P$ an accumulation point of $E$, the positive contingent of $E$ at $P$ is

$D^{+}(E: P)=\left\{y \in R^{n}:\right.$ there exists a sequence $\left\{P^{n}\right\} \subset E$ such that

$$
\text { as } \left.n \rightarrow \infty, P_{n} \rightarrow P, t_{n}>t_{p} \text {, and }\left|\left(x_{n}-x_{p}\right)\left(t_{n}-t_{p}\right)^{-1}-y\right| \rightarrow 0\right\} \text {. }
$$

The negative contingent and contingent of $E$ at $P$ are defined in a similar manner. If $E$ is the graph of a function $\varphi: R^{1} \rightarrow R^{n}$ and $P=\left(t_{0}, x_{0}\right)$ we write $D^{+} \varphi\left(t_{0}\right)$ instead of $D^{+}(E: P)$

A mapping $F: W \rightarrow c\left(R^{n}\right)$ is usc (upper semi-continuous) at $P \in W$ if, for each $\varepsilon>0$ there exists a $\delta>0$ such that $|Q-P|<\delta$ implies $r(F(Q)$, $F(P))<\varepsilon$. The mapping $F$ is $u s c$ on a set $E \subset W$ if it is usc at each point of the set. If, in this definition, we replace $r(F(Q), F(P))$ by $d(F(Q), F(P))$, then $F$ is continuous.

We shall consider the contingent equation

$$
x^{\prime} \in F(t, x) \text { where } F: W \rightarrow c c\left(R^{n}\right) \text { is use on } W \text {. }
$$

By a solution of (1) we mean a continuous function $\varphi: I \rightarrow R^{n}$, where $I$ is some interval in $R^{1}$, such that $D \varphi(t) \subset F(t, \varphi(t))$ for $t \in I$. (If $I$ contains end. points we use one sided contingents where necessary). It is known [13] that if $\varphi(t)$ is a solution of (1) on $I$, then $\varphi(t)$ is absolutely continuous and $\varphi^{\prime}(t) \in F^{\prime}(t, \varphi(t))$ a.e. on $I$.

The trajectory of a solution $\varphi(l)$, defined on $I$, is the set $\{(t, \varphi(t)): t \in I\}$. Later we discuss the maximal interval of existence of a solution $\varphi(t)$ which we shall denote by $D_{0}$.

We note that our results hold equally well for $W$ an open subset of $R^{\mathrm{s}} \times R^{n}$.

\section{2. - Basic Theory.}

ZaREMBA [16] proved that given $P \in W$ there exists a solution $\varphi(t)$ of (1) which is defined on some open interval containing $t_{p}$ and which satisfies $\varphi\left(t_{p}\right)=x_{p}$. (An account of Zaremba's work may also be found in [6]).

This theorem is the counterpart of the Peano existence theorem for or dinary differential equations. In developing the WAZEWSKI method in [3] we found it convenient to use not the Peano theorem, bat an existence theorem of NAGUMo [11]. The NAGUMo theorem translates to contingent equations. We include a proof for the sake of completenness. (The NAGumo theorem for differential inequalities, and a remark on contingent equations, may be found in [7]). 
Definimon. - A set $A \subset W$ is right admissible (with respect to (1)) if, for each $P \in A$ there exists an $a>0$ and a solution $\varphi(t)$ of (1) such that $\varphi(t)$ is defined and $(t, \varphi(t)) \in A$ for $t_{p} \leq t \leq t_{p}+a$ and $\psi\left(t_{p}\right)=x_{p}$.

Theonem 1. - A closed set $E \subset W$ is right admissible if and only if $D^{+}(E: P) \cap F(P) \neq \emptyset$ for all $P \in E$.

Proof. - That this condition is necessary for right admissibility is im. mediate.

We assume that in a neighborhood of $P, F(P)$ is continuous. If this is not the case, then in the manner of ZaREMBA (16, Theorem II.8] or [6, Theorem 1.3]), we may approximate $F$ by a family of such functions. We shall have the theorem for each member of the family and by a limiting argument the theorem holds for $F$.

We next observe that the following conditions are equivalent:

i) $D^{+}(E: P) \cap F(P) \neq \varnothing$ for all $P \in E ;$ ii) for $P \in E$ there exists a $v \in F(P)$ such that given $\varepsilon>0$ there exists a $Q \in E$ with $t_{p}<t_{Q}<t_{p}+\varepsilon$ and $\left|\left(x_{Q}-x_{p}\right)\left(t_{p}-t_{Q}\right)^{-1}-v\right|<\varepsilon ;$ iii $)$ for $P \in E$ and $\varepsilon>0$ there exists a $Q \in E$ with $t_{p}<t_{Q}<t_{p}+\varepsilon$ and $r\left(\left(\alpha_{Q}-x_{p}\right)\left(t_{Q}-t_{p}\right)^{-1}, F(P)\right)<\varepsilon$.

For $P_{0} \in E$ there exist a, $L>0$ such that $r(F(P), 0) \leq L$ on

$$
T=\left\{P: t_{0} \leq t_{p} \leq t_{0}+a,\left|x_{p}-x_{0}\right| \leq(L+1)\left|t_{p}-t_{0}\right|\right\} \text {. }
$$

For $\varepsilon, 0<\varepsilon<1$, let $\left\{P_{n}\right\}$ be a sequence of points $i$ n $E$ satisfying

$$
t_{n-1}<t_{n}<t_{n-1}+\varepsilon, r\left(\left(x_{n}-x_{n-1}\right)\left(t_{n}-t_{n-1}\right)^{-1}, F\left(P_{n-1}\right)\right)<\varepsilon
$$

$(n=1,2, \ldots)$ and $P_{0}$ given above. Let $M$ be the collection of all such points (for fixed $\varepsilon$ ) and let $b=\sup \{t:(t, x) \in M\}$.

If $b \leq t_{0}+a$, then $M$ has a limit point $(b, y) \in E \cap T$.

Hence, there exists a $(c, z) \in E$ such that

$$
b<c<b+\varepsilon, \quad r\left((z-y)(c-b)^{-1}, \quad F(b, y)\right)<\varepsilon .
$$

Now using the continity in $(b, y)$ of this last expression we can find a $P_{n} \in M$ such that

$$
t_{n}<c<t_{n}+\varepsilon, r\left(\left(z-x_{n}\right)\left(c-t_{n}\right)^{-1}, F\left(t_{n}, x_{n}\right)\right)<\varepsilon
$$

which contradicts the definition of $b$. So $b>t_{0}+a$.

For each integer $n>1$ there is a sequence $P_{0}, \ldots, P_{m}$ in $E$ such that $t_{m}>t_{0}+a$ and $t_{i-1}<t_{i}<t_{i-1}+\left(\frac{1}{n}\right)$,

$$
r\left(\left(x_{i}-x_{i-1}\right)\left(t_{i}-t_{i-1}\right)^{-1}, F\left(P_{i-1}\right)\right)<\left(\frac{1}{n}\right) \quad(i=1, \ldots, m) .
$$

Let $\varphi_{n}(t)$ be the polygonal function which joins $P_{0}, \ldots, P_{m}$. 
Then $\left|\varphi_{n}\left(t_{2}\right)-\varphi_{n}\left(t_{1}\right)\right| \leq(L+1)\left|t_{2}-t_{1}\right|$ so by the Ascoli theorem there exists a sub-sequence $\left\{\varphi_{k}(t)\right\}$ of $\left.\mid \varphi_{n}(t)\right\}$ which converges uniformly to $\varphi_{(t)}$ on $\left[t_{0}, t_{0}+a\right]$. Then $\varphi\left(t_{0}\right)=x_{0}, \varphi(t)$ is continuous (in fact absolutely continuous), and $(t, \varphi(t)) \in T \cap E$ for $t_{0} \leq t \leq t_{0}+a$. The lat ter is true since for $t \in\left[t_{0}, t_{0}+a\right]$, i) each $\left(t, \varphi_{n}(t)\right) \in T$ and $\left.i i\right)$ there is a sequence of points in $E$ which are vertices of the approximating polygonal lines which approach $(t, \psi(t))$.

The proof can be completed by showing that given $t \in\left[t_{0}, t_{0}+a\right]$ and $v>0$ there exists $\eta>0, N>0$ such that

$$
r\left(\left(\varphi_{k}(s)-\varphi_{k}(t)\right)(s-t)^{-1}, F(t, \varphi(t))\right)<2 \vee \text { for all } k \geq N
$$

$s \in\left[t_{0}, \dot{t}_{0}+a\right]$ with $0<|s-t|<\eta$. For then we can first let $k \rightarrow \infty$ and then $\vee \rightarrow 0$ and conclude that $D_{\varphi}(t) \subset F(t, \varphi(t))$.

Assume $t \in\left(t_{0}, t_{0}+a\right)$; the proof at the endpoints is similar. Since $F(P)$ is use there exists an $\eta, 0<2 \eta<\min \left(t-t_{0}, t_{0}+a-t\right)$ such that $r(F(u, x)$. $F(t, \varphi(t)))<\nu$ on $R=\{(u, x):|u-t| \leq 2 \eta,|x-\varphi(u)| \leq \eta\}$. Choose $N$ such that $k \geq N$ implies $i)(1 / k)<v$, ii $)\left|\varphi_{k}(u)-\psi(u)\right| \leq \eta$ for $|u-t| \leq 2 \eta$, and iii) $(1 / k)<\eta$ (so that if $P_{0}, \ldots, P_{m}$ are the vertices of $\varphi_{k}(t)$, then $\max$ $\left.\left\{\left(t_{i}-t_{i-1}\right): i=1, \ldots, m\right\}<\eta\right)$. Then $k \geq N,|u-t| \leq \eta$ implies $r\left(F\left(u, \varphi_{k}(u)\right)\right.$, $F(t, \varphi(t))<\psi$. Now $D \xi_{k}(u)=\left(x_{j}-x_{j-1}\right)\left(t_{j}-t_{j-1}\right)^{-1}$ where $P_{j-1} \in R$ if $|u-t| \leq \eta$. So $r\left(D \varphi_{h}(u), F(t, \varphi(t))\right) \leq r\left(D \varphi_{k}(u), F\left(P_{j-1}\right)\right)+r\left(F\left(P_{j-1}\right), F(t, \varphi(t))\right)<2 v$ for $|u-l| \leq \eta$, $k \geq N$.

If $\psi(t)$ is a continuous function defined on $[d, e]$ and if $D \psi(b) \subset A$, a nonempty, convex set, for $d \leq t \leq e$, then $(\bigcup(d)-\psi(e))(d-e)^{-1} \in A$. (See [16, Theorem II.5] or [1]).

Since $S=\{x: r(x, F(t, \varphi(t))) \leq 2 v\}$ is compact and convex and since $D \varphi_{k}(u) \subset S$ for $k \geq N, 0<|u-t|<|s-t|$, we can conclude that $\left(\varphi_{k}(s)-\right.$ $\left.-\varphi_{k}(t)\right)(s-t)^{-1} \in S$ for $k \geq N, 0<|s-t|<\eta$.

RemaRKs. - 1) As in the theory of ordinary differential equations, any solution $\varphi(t)$ of (1) can be extended to a maximal interval of existence which we shall denote by $D_{\varphi}$.

2) If $E \subset W$ is locally compact and $F(P)$ is defined and usc only on $E$, the theorem still holds. For then $E$ is relatively closed in some open $U \subset W$, $F$ has a usc extension to all of $U$, and the preceding applies.

3) If $F$ is defined and usc on a locally compact subset $E \subset W$, if $E$ is right admissible, if $\varphi(t, P)$ is a solution of (1) passing through $P \in E$, and if $(t, \varphi(t, P)) \rightarrow Q \in \operatorname{Fr}(E)$ as $t \rightarrow \operatorname{Fr}\left(D_{\varphi}\right)$, then $Q \notin \operatorname{Fr}(E) \cap E$. (For remarks on the continuation of solntions see [2]).

The next definition and two theorems follow Yonke [14], [15]. 
Definitron. - A set $A \subset W$ is positively weakly invariant (with respect to (1)) if and only if for each $P \in A$ there exists a solution $\varphi(l)$ of (1) such that $\varphi\left(t_{p}\right)=x_{p}$ and $(t, \varphi(t)) \in A$ for $t \in D_{\varphi} \cap\left[t_{p}, \infty\right)$. Negatively weakly invariant and weakly invariant are delined in a similar manner.

Theonem 2. - a) A set $A \subset W$ is weakly invariant if and only if it is a union of a family of trajectories of solutions of (1). b) A closed set $E \subset W$ is positively weakly invariant if and only if it is right admissible and hence if and only if $D^{+}(E: P) \cap E(P) \neq \emptyset$ for all $P \in E$.

Proof. - The proof is straightforward.

Theorem 3. - If $G_{1}, G_{2} \subset W$ are closed and positively weakly invariant and if $W=G_{1} \cup G_{2}$, then $H=G_{1} \cap G_{2}$ is positively weakly invariant.

Proof. - Assume $H$ is not positively weakly invariant and let $P_{0} \in H$. Then there exists two trajectories $\varphi_{i}(t)$ of $(1)$ with $\left.\varphi_{i}^{\prime} t_{0}\right)=x_{0}(i=1,2)$ and an $a>0$ such that $\left(t, \varphi_{i}(t)\right) \in G_{i}$ for $t_{0} \leq t<t_{0}+a, i=1$, 2. Let $L(t)$ be the segment joining $\left(t, \varphi_{1}(t)\right)$ to $\left(t, \varphi_{2}(t)\right)$ and let $(t, x(t)) \in L(t) \cap\left(G_{1} \cap G_{2}\right)$. Here we use the restrictions placed on $G_{1}, G_{2}$.

Then $x(t)=\alpha(t) p_{1}(t)+(1-\alpha(t)) p_{2}(t), 0 \leq \alpha(t) \leq 1$.

Choose a sequenee $\left\{t_{n}: t_{n} \rightarrow t_{0}+\right.$, and $\left.\alpha\left(t_{n}\right) \rightarrow \alpha_{0}\right\}$. Then

$$
\begin{gathered}
\left(x\left(t_{n}\right)-x_{0}\right)\left(t_{n}-t_{0}\right)^{-1}=\left(\alpha\left(t_{n}\right)\left(\varphi_{1}\left(t_{n}\right)-x_{0}\right)+\left(1-\alpha\left(t_{n}\right)\right)\right. \\
\left.\left(\varphi_{2}\left(t_{n}\right)-x_{0}\right)\right)\left(t_{n}-t_{0}\right)^{-1} .
\end{gathered}
$$

We can now choose a subsequence $\left\{t_{k}\right\}$ of $\left\{t_{n}\right\}$ such that in the limit the left side of this expression belongs to $D^{+}\left(H: P_{0}\right)$ and the right side equals $\alpha_{0} v_{1}+\left(1-\alpha_{0}\right) v_{2}$ where $v_{i} \in D^{+} \varphi_{i}\left(t_{0}\right)(i=1,2)$.

By convexity this vector is in $F\left(P_{0}\right)$ hence $D^{+}\left(H: P_{0}\right) \cap F\left(P_{0}\right) \neq \emptyset$ and $H$ is positively weakly invariant.

In the sequel we shall use the following KaMKE-type convergence theorem. (See for example [8, theorem 3.2]).

Theorem 4. - Let $\left\{P_{n} \in W: P_{n} \rightarrow P_{0}\right.$ as $\left.n \rightarrow \infty\right)$ and let $\varphi_{n}(t)$ be a solution of (1) with $\varphi_{n}\left(t_{n}\right)=x_{n}$. Then there exists a solution $\varphi(t)$ of (1) with $\varphi\left(t_{0}\right)=x_{0}$ and with maximal interval of existence $D_{\varphi}$ and a subsequence $\left\{\varphi_{n}(t)\right\}$ of $\left\{\varphi_{n}(t)\right\}$ such that for any compact subinterval $I \subset D_{\varphi}$ and all $k$ sufficiently large, $\varphi_{k}(t)$ is defined on $I$ and $\varphi_{k}(t) \rightarrow \varphi^{(t)}$ as $k \rightarrow \infty$ uniformly on $I$.

Proof. - Let $\varphi_{n}(t)$ be as in the hypotheses. There exists a, $N>0$ such that if $n \geq N$, then $\varphi_{n}(t)$ is defined and uniformly bounded for $\left|t-t_{0}\right| \leq \alpha$. Just let $R=\left\{(t, x):\left|t-t_{0}\right| \leq 2 b,\left|x-x_{0}\right| \leq 2 b\right\}$ for some $b>0, M=\max$ $\{r(F(P), 0): P \in R\}$, and choose $a=\min ((b / 2),(b / 2 M))$ and $N$ such that $n \geq N$ implies $\left|P_{n}-P\right|<b$. 
If $\left\{\varphi_{n}(t)\right\}$ is a sequence of solutions of (1) which are defined, uniformly bounded, and equicontinuous on an interval $I$, then exists a subsequence $\left\{\varphi_{k}(l)\right.$ ) such that $\varphi_{k}(t) \rightarrow \psi(t)$ uniformly on $I$ as $k \rightarrow \infty$ (by the ArzeLA-Ascoli theorem) and $\psi(t)$ is a solution of (1) (by ZAREMBA, [16, theorem II.6]).

Let $\left\{\varphi_{n}(l)\right\}$ be as in the hypotheses and choose a as in the first paragraph of the proof. Let $\left\{\varphi_{k}(t)\right\}$ be a subsequence of $\left\{\varphi_{n}(t)\right\}$ such that $\varphi_{k}(t) \rightarrow \psi(t)$ uniformly on $\left|t-t_{0}\right| \leq a$ where $\psi(t)$ is a solution of $(1)$ with $\psi\left(t_{0}\right)=x_{0}$.

Let $(b, c)$ be the maximal interval such that there exists a solution $\varphi(t)$ defined on $(b, c) ; \varphi(t)=\psi(t)$ on $\left|t-t_{0}\right|<a$; and for each oompact $I \subset(b, c)$, some subsequence of $\left\{\varphi_{k}(t)\right\}$ is defined and converges to $\varphi(t)$ on $I$.

We elaim that $\varphi(t)$ is not defined at $t=b, c$ and hence $(b, c)=D_{0}$ and $\varphi$ satisfies the conclusion of the theorem. For suppose $\varphi(b)$ is defined. We can find a subsequence of $\left\{\varphi_{h}(t)\right\}$ and a sequence $\left\{t_{m}: t_{m} \rightarrow b-\right\}$ such that $\left|\varphi_{m}(t)-\varphi(t)\right|<\left(\frac{1}{m}\right)$ on $\left[t_{0}, t_{m}\right]$. Now using the first two paragraphs of the proof we can extend $\varphi(t)$ beyond $b$.

\section{3. - The consequent mapping and Wazewski's theorem.}

Definitions. - Let $V$ be an open subset of $W$. A point $Q \in \operatorname{Fr}(V)$ is a consequent of a point $P \in V$ (relative to (1)) if $t$ ere exists a solution $\varphi(t, P)$ of (1) and a $t_{1}, t_{p}<t_{1} \leq t_{Q}$, such that $\varphi(t, P)$ is defined on $\left[t_{p}, t_{Q}\right],(t, \varphi(t, P)) \in V$ for $t_{p} \leq t<t_{1},(t, \varphi(t, P)) \in \operatorname{Fr}(V)$ for $t_{1} \leq t \leq t_{Q}$, and $\left(t_{Q}, \varphi\left(t_{Q}, P\right)\right)=Q$. The point $Q \in \operatorname{Fr}(V)$ is a consequent of a point $P \in \operatorname{Fr}(V)$ (and $P$ may equal $Q$ ) if there exists a solution $\varphi(t, P)$ of $(1)$ such that $(t, \varphi(t, P)) \in \operatorname{Fr}(V)$ for $\left[t_{P}, t_{Q}\right]$. Consequents will also be called points of egress and the set of all egress points will be denoted by $S$.

A point $Q \in S$ is a strict egress point if for every solution $\varphi(t, Q)$, $c_{q}=\sup \left(t:(s, p(s, Q)) \in F r(V), t_{Q} \leq s \leq t\right)<\infty$ and there exists a sequence $\left\{t_{n}: t_{n} \rightarrow c_{\vartheta^{+}}\right\}$with $\left(t_{n}, \varphi\left(t_{n}, Q\right)\right) \in W-\bar{V}$. The mapping $C:(V \cup S) \rightarrow \operatorname{Fr}(V)$ defined by $O(P)=\{Q \in F r(V): Q$ is a consequent of $P \mid$ is the consequent mapping.

A solution $\varphi(t, P), P \in V$, leaves $V$ if there exists some $t_{1} \in D_{\varphi} \cap\left[t_{P}, \infty\right)$ such that $\left(t_{1}, \varphi\left(t_{1}, P\right)\right) \in W-V$.

Theonem 5. - Let $C:(V \cup S) \rightarrow F r(V)$ be the consequent mapping and let $P \in V \cup S$. If all solutions through $P$ leave $V$ and if all points of egress are strict, then $C(P)$ is compact.

Proof. - We shall show that any sequence $\left\{Q_{n}\right\} \subset C(P)$ contains a subsequence which converges to a point of $C(P)$. 
Let $\varphi_{n}(t)$ be a solution of (1) through $P$ such that $\left(t_{n}, \varphi_{n}\left(t_{n}\right)\right)=Q_{n}$. By Theorem 4 there is a subsequence $\left\{\varphi_{k}(l)\right.$ ! of $\left\{\varphi_{n}(t)\right\}$ which converges to a solation $\varphi(t)$ of (1) with $\varphi\left(t_{p}\right)=x_{p}$. By assumption, $\varphi(t)$ leaves $V$ and every point of egress is strict.

Hence there exists a $t_{a}>t_{p}$ such that $\left(t_{a}, \varphi\left(t_{a}\right)\right) \in W-\bar{V}$. For all $k$ suffieiently large, $\left(t_{a}, \varphi_{k}\left(t_{a}\right)\right) \in W-\bar{V}$ hence $t_{p}<t_{k}<t_{a}$. Choose a subsequence $\left\{t_{m}\right\}$ of $\left\{t_{k}\right\}$ such that $t_{m} \rightarrow t_{b}<t_{a}$. Them $Q_{m}=\left(t_{m}, \varphi_{n}\left(t_{m}\right)\right) \rightarrow\left(t_{b}, \varphi\left(t_{b}\right)\right)$.

Now $\left(t_{b}, \varphi\left(t_{b}\right)\right) \notin C(P)$ implies that for $m$ sufficiently large, $Q_{m}$ is pot an egress point. The possibilities (for example, $\left(t_{c}, \varphi\left(t_{c}\right)\right) \in W-\bar{V}$ for some $\left.t_{c} \in\left(t_{P}, t_{b}\right)\right)$ are easily checked. Thus $O(P)$ is compact.

Theorem 6. - Let $C:(V \cup S) \rightarrow \operatorname{Fr}(V)$ be the consequent mapping and let $I^{\prime} \in V \cup S$. If all solutions through $P$ leave $V$ and all points of egress are strict, then $C(P)$ is connected.

Proof. - If $P \in S$, the proof is immediate. So assume $P \in V$.

If $C(P)$ is not connected, then $C(P)=C_{1} \cup C_{2}$ where $C_{1}$ and $C_{2}$ are disjoint compact subsets of $W$.

For $\varphi(t, P)$ a solution of (1) with $P \in V$ let $D_{\varphi}=(\alpha, \omega)$ be the maximal interval of existence of $\varphi(t, P)$ relative to $W$ and let $E_{0}=(\beta, \gamma)$ be the maximal interval of existence of $\varphi(t, P)$ relative to $V$, i.e. $E_{p}$ is the largest open interval about $t_{p}$ such that $(t, \varphi(t, P)) \in V$ for $t \in E_{\varphi}$. Let $\Phi(P)$ denote the trajectory of $\varphi(t, P)$ relative to $V$, i.e. $\Phi(P)=\left\{(t, \varphi(t, P)): t \in E_{0} !\right.$. Let $\sigma(A, B)=\inf \{|a-b|: a \in A, b \in B\}$.

Let $R=\left\{\Phi(Q): Q \in V\right.$ and $\left.\sigma\left(\Phi(Q), C_{1}\right) \leq \sigma\left(\Phi(Q), C_{2}\right)\right\}$ and let $S$ be a set similarly defined but with the inequality reversed. Then $R, S$ are positively weakly invariant and closed, relative to $V, V=R \cup S$, and $P \in R \cap S$. We may conclude by Theorem 8 that there exists a $\varphi(t, P)$ such that $(t, \varphi(t, P)) \in R \cap S$ for $t_{p} \leq t<\gamma$.

Since $\varphi(l, P)$ leaves $V, \gamma<\omega$ and $(\gamma, \varphi(\gamma, P)) \in F r(V)$, say $(\gamma, \varphi(\gamma, P)) \in C_{1}$. Then $0=\sigma\left(\Phi(P), C_{1}\right)=\sigma\left(\Phi(P), C_{2}\right)>0$ and from this contradiction we conclude that $C(P)$ is connected.

Theonem 7. - Let $C:(V \cup S) \rightarrow \operatorname{Fr}(V)$ be the consequent mapping and let $A$ be a nonempty subset of $V \cup S$. If all solutions through $P$ leave $V$, for each $P \in A$, and if all points of egress are strict, then $C$ is use on $A$.

Proof. - By Theorem 5, $C(P)$ is eompact for each $P \in A$.

If $C$ is not use at some $P_{0} \in A$, then there exists an $\varepsilon>0$ and a sequence $\left\{P_{n} \in A: P_{n} \rightarrow P_{0}\right.$ and $r\left(C\left(P_{n}\right), C\left(P_{0}\right)\right) \geq \varepsilon$ as $\left.n \rightarrow \infty\right\}$.

Hence, for $\mathrm{r}_{d i}$ each $n$, there is a $Q_{n} \in C\left(P_{n}\right)$ such that $r\left(Q_{n}, C\left(P_{0}\right)\right) \geq \varepsilon$. Let $\varphi_{n}(t)$ be a solution of (1) through $P_{n}$ and $Q_{n}$. By Theorem 4 there is a 
subsequence $\left\{\varphi_{k}(t)\right\}$ of $\left.\left\{\varphi_{n}{ }^{\prime} t\right)\right\}$ which converges to a solution $\varphi(t)$ of (1) with $\varphi\left(t_{0}\right)=x_{0}$. By assumption, $\varphi(t)$ leaves $V$ and every point of egress is strict. Now just as in the proof of Theorem 5 we can find a subsequence $\left\{t_{m}\right\}$ of $\left\{t_{k}\right\}$ such that $\left(t_{m}, \varphi_{m}\left(t_{m}\right)\right)=Q_{m} \rightarrow\left(t_{b}, \varphi\left(t_{b}\right)\right) \in C\left(P_{0}\right)$ which is a contradiction.

Remark. - If $A$ is a compact and connected subset of $V \cup S$, then under the hypotheses of Theoren $7, C(A)$ is compact and connected. This is a consequence of the properties of use functions. (See for example [3], Theorem 1).

Definition. - Let $A, B$ be subsets of $R^{n+1}$ with $B \subset A$. If there exists a usc mapping $G: A \rightarrow c\left(R^{n+1}\right)$ such that $G(x) \subset B$ and $G(x)$ is connected for all $x \in A$ and $x \in G(x)$ for all $x \in B$, then $B$ is a set-valued retract of $A$ and $G$ is a sel-valued retraction from $A$ into $B$.

Theorem 8. - If there exists a set $Z \subset V \cup S$ such that $Z \cap S$ is a set-valued retract of $S$ but not of $Z$ and if all points of $S$ are points of strict egress, then there exists a $P \in Z$ and a solution $\varphi(t, P)$ of (1) such that $(t, \varphi(t, P)) \in V$ for $t \in D_{\varphi} \cap\left[t_{P}, \infty\right)$.

Proof. - If, for all $P \in Z$, every solution $\varphi(t, P)$ of (1) leaves $V$, then the consequent mapping $C: Z \rightarrow S$ is usc.

Let $H: S \rightarrow Z \cap S$ be the set-valued retraction which is given in the hy potheses. Then $H C: Z \rightarrow Z \cap S$ is usc and for each $P \in Z \cap S, P \in H(P) \subset H C(P)$. Thus $Z \cap S$ is a set-valued retract of $Z$ which is a contradiction.

The authors would like to acknowledge several helpful discussions with $S$. Sedziwy during the preparation of this paper.

\section{REFERENCES}

[1] E. A. Barbashin and Yu. I. ALimov, On the theory of real differential equations (in Russicn), Izv. Vyss. Ucebn. Zaved. Matematika 1 (26) (1962), 3-13.

[2] J. Bebernes, W. Fulks, and G. Meisters, Differentiable paths and the continuation of solutions of differential equations, J. Differential Equations 2(1966), 102.106.

[3] J.W. Bebernes and J.D. SchuUR, Investigations in the topological method of Waze. wski, Submitted.

[4] A. BцLеоKr, Sur une méthode de régularization des équations différentielles ordinaires dont les intëgrales ne remplissent pas la condition "d' unicité, Bull. Acad. Polon. Sci. 4 (1956), 497.501 .

[5] A. BIELECKX and C. KCUCZNY, Sur une théoreme concernant des systèmes d'équations differentielles ordinaires, Ann. Univ. Mariae Curie.Sklodowska, Sect. A. 14, 8(1960), 115.123. 
[6] T. F. BRIdgLand, Jr., Contributions to the theory of generalized differential equations $I$, Math. Systems Theory $3(1969), 17.50$.

[i] M. Hukunara, Sur un théorème de Kneser, J. Fac. Sci. Univ. Tokyo, Sect. I 6 (1953), 329-344.

[8] P. Hartman, Ordinary Differential Equations, John Wiley, 1964.

[9] L. JACKSON and G. KLAASEN, A variation of Wazenski's topotogical method, SIAM J. Appl. Math. To appear.

[10] C. KLUCZNy, Sur certaines familles de courbes en relation avec la theorie des differen. tielles ordinaires I, II. Ann. Univ. Mariae Curie.Sklodowska, Sect. A 15(1960), 13-40, $16(1962), 5-18$.

[11] М. NAGUMo, Über die Lage der Integralkurven genöhnlicher Differentialgleichungen, Proc. Phys.-Math. Sne. Japan 24 (1942), 551.อ๊59.

[12] T. WAZEWSKI, Une methode topologique de l'examen du phénomène asymptotique rela. tivement aux équations différentielles ordinaires, Rend. Accad. Lincei (8) 3 (1947), $210 \cdot 215$.

[13] -- -, Sur une condition équitalente à l'équation au contingent, Bull. Acad. Polon. Sci. Sex. Math. Astr. Phys. 9 (1961), 865.867.

[14] J.A. Yonke, Invariance for ordinary differeutial equations, Math. Systems Theory 1 (1967), 35̄3-372.

[15] - - Invariance of Contingent Fquations, Lecture Notes in Operations Research and Mathematical Economics 12: Mathematical Systems Theory and Economics II. Sprin. ger-Verlag, 1969.

[16] S. C. Zaremba, Sur les équations au paratingent, Bull. Sei Math. (2) 60 (1936), 139.160. 